Journal of Applied Fluid Mechanics, Vol. 9, Special Issue 1, pp. 173-176, 2016.

Selected papers from the $7^{\text {th }}$ International Exergy, Energy

and Environment Symposium, IEEE7-2015

Available online at www.jafmonline.net, ISSN 1735-3572, EISSN 1735-3645.

DOI: $10.36884 /$ jafm.9.SI1.25818

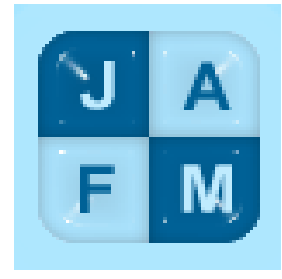

\title{
Numerical Investigation of Non-Newtonian Blood Effect on Acoustic Streaming
}

\author{
R. Aayani ${ }^{1}$, A. Shahidian ${ }^{2 \dagger}$ and M. Ghassemi ${ }^{3}$ \\ ${ }^{1}$ Department of Mechanical Engineering, K.N.Toosi University of Technology,Tehran, 1999143344, Iran \\ ${ }^{2}$ Department of Mechanical Engineering, K.N.Toosi University of Technology,Tehran, 1999143344, Iran \\ ${ }^{3}$ Centre for Hydrogen and Fuel Cell Research, University of Birmingham, Birmingham, B15 2TT, UK (on \\ sabbatical from K. N. Toosi University of Technology)
}

†Corresponding Author Email:shahidian@kntu.ac.ir

(Received October 20, 2015; accepted December 10, 2015)

\begin{abstract}
Acoustic streaming, as an important phenomenon, is used in a wide variety of applications such as drug delivery and the removal of plaque in the vein surfaces. The purpose of the current paper is to investigate the effect of blood, as a non-Newtonian fluid, on acoustic streaming. The governing non-linear differential equations, mass, momentum, and state equations for non-stationary fluid using second-order perturbation theory, are coupled and solved. An in house computational fluid dynamics (CFD) code based on the finite element method is utilized. Results show that viscosity is highly dependent on shear stresses, about $60 \%$. In addition viscosity affects the acoustic streaming velocity field.
\end{abstract}

Keywords: "Non-Newtonian; Blood viscosity; Acoustic streaming.

\section{NOMENCLATURE}

$\begin{array}{ll}p & \text { pressure } \\ T & \text { matrix transpose } \\ \mathrm{t} & \text { time } \\ \mu & \text { viscosity }\end{array}$

\section{INTRODUCTION}

Ultrasonic waves have a variety of applications such as the diagnosis and treatment of diseases, drug delivery, and cell separation. As Ultrasonic waves pass through objects including tissues and organs. When used in tissues and organs, it leads to chemical, physical and biological changes. When ultrasonic waves pass through human body, heat, bubbles, stress and vibration areproduced; this can be beneficial or harmful.

To reduce the hazard to health and improve the efficiency of acoustic wave, many researches have been conducted to manage the applied ultrasonic field. "Solovchuk et al. (2012)" investigated the influence of blood vessels on temperature distribution during high-intensity focused ultrasound (HIFU) ablation of liver tumors. They coupled a three-dimensional acoustics-thermal-fluid model based on the linear Westervelt, bioheat and Navier-Stokes equations and solved to compute the

\author{
$v$ velocity \\ $\rho$ density \\ $\omega$ angular velocity \\ $\dot{\gamma}$ shear rate
}

temperature field in the hepatic cancerous region. Also Solovchuk et al. showed that acoustic streaming significantly change the temperature in a large blood vessel. "Bernassau et al. (2014)" assessed the acoustic streaming in a multitransducer quasi- standing wave acoustic particle manipulation device. They experimentally observed that the streaming takes the form of two main vortices that have their highest velocity in the region where the standing wave is established. Bernassau et al. developed a finite element model that agrees with experimental results. They showed that the Reynolds stresses, that give rise to the fluid motion, are strongest in the high velocity region. The effects of temperature dependence of viscosity and density on the acoustic radiation force and the boundary-driven acoustic streaming in microchannel acousto fluidics investigated by "Muller and Bruus (2014)". They calculated the acoustic streaming slip velocity for the bulk flow for the case of an ultrasound wave scattering on a compressible, spherical particle suspended in a viscous, thermal conducting fluid. Muller and Bruus 
included the viscosity and the volume thermal expansion coefficient of the fluid and derived an analytical expression for the radiation force.

As known, viscosity varies in non-Newtonian fluid such as blood. It also plays a major role in acoustic streaming. Therefore, the purpose of the current study is to investigate the effect of blood as a nonNewtonian fluid on acoustic streaming in a blood vessel. To determine the effect of viscosity on acoustic streaming, the couple governing non-linear differential equations, mass, momentum, and state equations for non-stationary fluid using secondorder perturbation theory, are solved. An in house computational fluid dynamics (CFD) code based on the finite element method is utilized.

\section{PROPOSE MODEL}

\subsection{Geometry and boundary conditions}

Schematic of the geometry used in this study is depicted in Fig.1. As shown, it is a 2-D channel with a $6 \mathrm{~mm}$ by $1 \mathrm{~mm}$ that resembles the coronary arterial in a human body is utilized. Blood with mean velocity $6 \mathrm{~mm} / \mathrm{s}$ flows into the vessel from the left side of the channel and exists at right side. The bottom wall of the channel vibrates due to ultrasonic wave propagation. Therefore it is modeled by corresponding velocity of vibration. It is assumed that a half wavelength standing wave of $750 \mathrm{KHz}$ frequency is developed. As a result the top wall of the channel is assumed to vibrate as well.

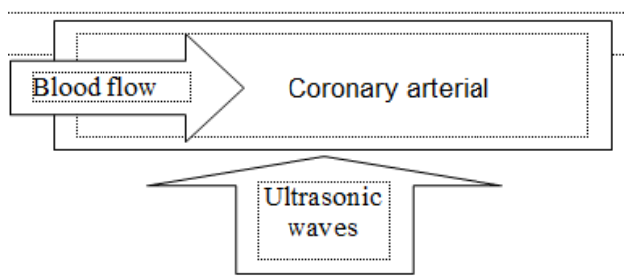

Fig. 1. Schematic of geometry.

\subsection{Governing equations}

As mentioned in table 1, Second-order perturbation theory is applied for pressure, velocity and density, given in equations (1), (2) and (3). Second order perturbations are main source non-linearity in the governing equations. It is noted that mean time integration of v2 is the acoustic streaming and time integration of v1 equals zero. Continuity and momentum equations for zero order are stated by equations (4) and (7), respectively. Also, the first order and second of continuity and momentum equation are given by equations (5), (8), (6) and (9) respectively. Since blood is assumed to be nonNewtonian, the viscosity in momentum equations, (7) - (9), is based on power law and is given by equation (10).

\section{NUMERICAL SCHEME}

In order to solve the non-linear governing differential equation, an in house numerical code
Table 1 governing equations

\begin{tabular}{|c|c|}
\hline$p=p_{0}+p_{1}+p_{2}$ & 1 \\
\hline$\vec{v}=\vec{v}_{0}+\vec{v}_{1}+\vec{v}_{2}$ & 2 \\
\hline$\rho(p)=\rho_{0}+\rho_{1}+\rho_{2}$ & 3 \\
\hline$\frac{\partial \rho_{0}}{\partial t}=-\nabla \cdot\left(\rho_{0} \vec{v}_{0}\right)$ & 4 \\
\hline$\frac{\partial \rho_{1}}{\partial t}=-\nabla \cdot\left(\rho_{0} \vec{v}_{1}+\rho_{1} \vec{v}_{0}\right)$ & 5 \\
\hline$\frac{\partial \rho_{2}}{\partial t}=-\nabla \cdot\left(\rho_{0} \vec{v}_{2}+\rho_{1} \vec{v}_{1}+\rho_{2} \vec{v}_{0}\right)$ & 6 \\
\hline $\begin{array}{l}\rho_{0} \frac{\partial \vec{v}_{0}}{\partial t}=-\nabla p_{0}-\rho_{0}\left(\vec{v}_{0} \cdot \nabla\right) \vec{v}_{0} \\
+\nabla \cdot\left(\mu_{0}\left(\nabla \vec{v}_{0}+\nabla \vec{v}_{0}^{T}\right)\right)+\nabla\left(\lambda_{0}\left(\nabla \cdot \vec{v}_{0}\right)\right)\end{array}$ & 7 \\
\hline $\begin{array}{l}\rho_{0} \frac{\partial \vec{v}_{1}}{\partial t}+\rho_{1} \frac{\partial \vec{v}_{0}}{\partial t}=-\nabla p_{1}-\rho_{0}\left(\vec{v}_{1} \cdot \nabla\right) \vec{v}_{0} \\
-\rho_{0}\left(\vec{v}_{0} \cdot \nabla\right) \vec{v}_{1}-\rho_{1}\left(\vec{v}_{0} \cdot \nabla\right) \vec{v}_{0} \\
+\nabla \cdot\left(\mu_{0}\left(\nabla \vec{v}_{1}+\nabla \vec{v}_{1}^{T}\right)\right) \\
+\nabla \cdot\left(\mu_{1}\left(\nabla \vec{v}_{0}+\nabla \vec{v}_{0}^{T}\right)\right) \\
+\nabla\left(\lambda_{0}\left(\nabla \cdot \vec{v}_{1}\right)\right)+\nabla\left(\lambda_{1}\left(\nabla \cdot \vec{v}_{0}\right)\right)\end{array}$ & 8 \\
\hline $\begin{array}{l}\rho_{0} \frac{\partial \vec{v}_{2}}{\partial t}+\rho_{1} \frac{\partial \vec{v}_{1}}{\partial t}+\rho_{2} \frac{\partial \vec{v}_{0}}{\partial t}=-\nabla p_{2} \\
-\rho_{0}\left(\vec{v}_{0} \cdot \nabla\right) \vec{v}_{2}-\rho_{0}\left(\vec{v}_{1} \cdot \nabla\right) \vec{v}_{1}-\rho_{0}\left(\vec{v}_{2} \cdot \nabla\right) \vec{v}_{0} \\
-\rho_{1}\left(\vec{v}_{0} \cdot \nabla\right) \vec{v}_{1}-\rho_{1}\left(\vec{v}_{1} \cdot \nabla\right) \vec{v}_{0}-\rho_{2}\left(\vec{v}_{0} \cdot \nabla\right) \vec{v}_{0} \\
+\nabla \cdot\left(\mu_{0}\left(\nabla \vec{v}_{2}+\nabla \vec{v}_{2}^{T}\right)\right) \\
+\nabla \cdot\left(\mu_{1}\left(\nabla \vec{v}_{1}+\nabla \vec{v}_{1}^{T}\right)\right) \\
+\nabla\left(\lambda_{0}\left(\nabla \cdot \vec{v}_{2}\right)\right)+\nabla\left(\lambda_{1}\left(\nabla \cdot \vec{v}_{1}\right)\right)\end{array}$ & 9 \\
\hline$\mu=K \dot{\gamma}^{n-1}$ & 10 \\
\hline
\end{tabular}

based on finite element method is utilized. 5000 quad mapped meshes are generated and applied. First, zero order continuity and momentum equations, Eqs. (4) and (7), are solved and the steady state solution of $\mathrm{p} 0$ and $\mathrm{v} 0$ are obtained. Then Eqs (5) and (8) are transferred to frequency domain in order to avoid transient solution. Finally Eqs. (6) and (9) are time averaged and solved to obtain acoustic streaming velocity profile.

\section{RESULTS AND DISCUSSSIONS}

The viscosity as a function of shear stress for the 
entire channel is depicted in Fig 2. As shown wall shear stress for Newtonian and non-Newtonian fluid are $36(1 / \mathrm{s})$ and $41(1 / \mathrm{s})$, respectively. These values of viscosity justify our assumption of blood as nonNewtonian fluid in selected channel.

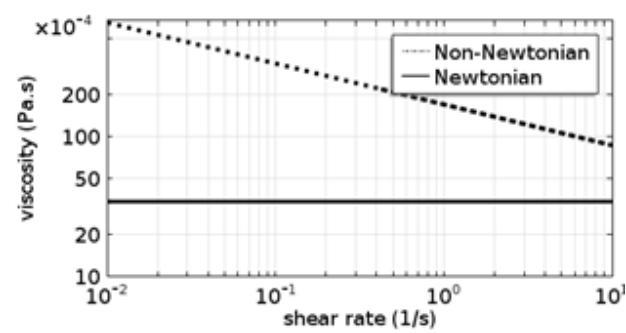

Fig. 2. Viscosity as a function of shear rate.

Figures 3 and 4 depict the zero-order $\mathrm{x}$-velocities profiles at vertical and horizontal sections respectively. As shown, the trend for the velocity of the Newtonian and non-Newtonian fluid is the same. However the magnitude of Newtonian fluid velocity is higher than non-Newtonian fluid. This is due to the shear rate value which is less than 100 . Also non-Newtonian model reaches its fully developed velocity at a point closer to entrance.

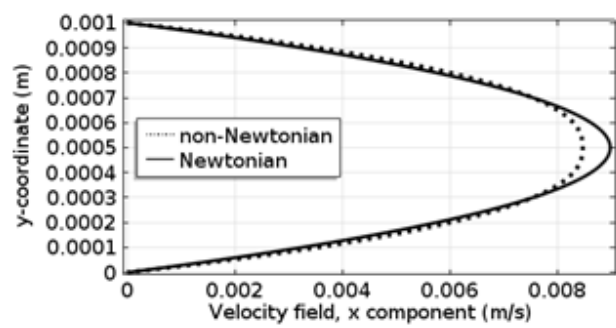

Fig. 3. $x$-velocity profile at vertical section.

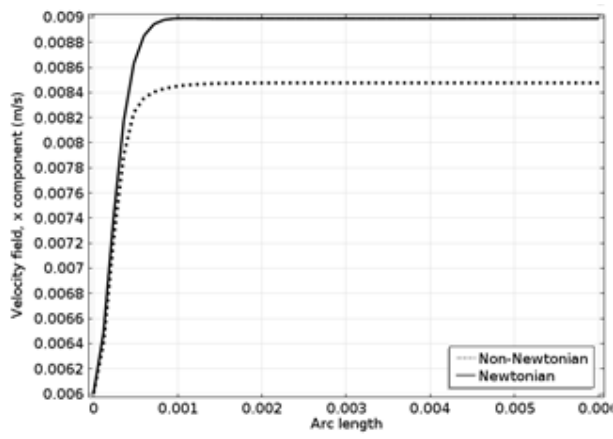

Fig. 4. $x$-velocity profile at middle height of channel.

First-order $\mathrm{x}$-velocity profile at the middle height of the channel is depicted in Fig. 5. As shown, again, the trend for Newtonian and non-Newtonian fluid is the same for first order perturbations velocity.

Figure 6 shows the second-order velocity of the Newtonian model. Again, the velocity trend for both fluids are same, while the velocity magnitude

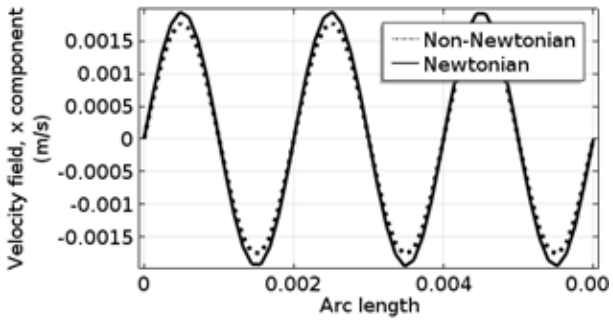

Fig. 5. First order $x$-velocity velocity profile at middle height.

of Newtonian is more than twice of the nonvelocity, acoustic streaming velocity, is highly dependent on the viscosity model of the blood. Increasing ultrasonic frequency or intensity, amplifies acoustic streaming velocity.

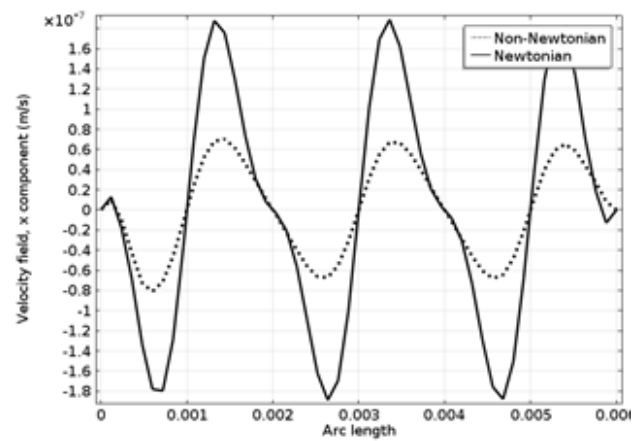

Fig. 6. Second order $x$-velocity velocity profile at middle height.

Newtonian fluid. As expected the second-orderThe total $\mathrm{x}$-velocity profile at the middle height of the channel is shown in Fig.7.

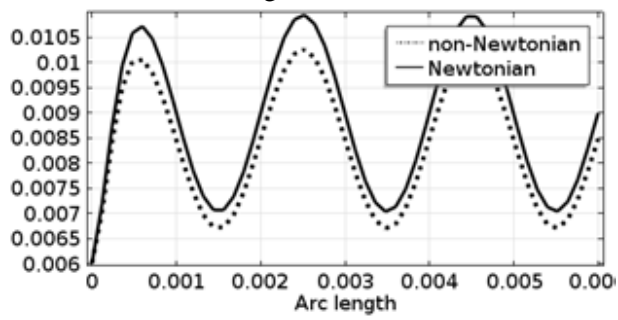

Fig. 7. Total x-velocity profile at middle height.

As shown by applying acoustic field the steadystate velocity is changed to a pulsatile velocity profile.

Also the $750 \mathrm{KHz}$ acoustic field causes a sinusoidal velocity just as like as a non-steady pulsatile flow by 180 (bpm), in comparison with blood velocity before applying acoustic field as shown in Fig.4. As blood flow passes the vessel at mean velocity of $6(\mathrm{~mm} / \mathrm{s})$ and one oscillation in velocity occurs at each $2(\mathrm{~mm})$, the 180 beats a minute is reached. It means that at 1 second, blood velocity oscillates 3 times.

Figures 8 and 9 depict the acoustic streaming inner 
and outer boundary layer for non-Newtonian and Newtonian viscosity model respectively.

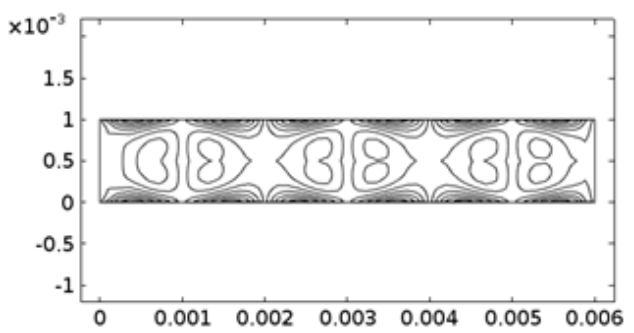

Fig. 8. Acoustic streaming inner and outer boundary layer for non-Newtonian viscosity model.

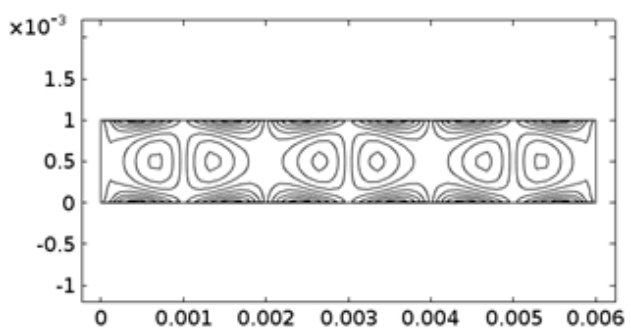

Fig. 9. Acoustic streaming inner and outer boundary layer for Newtonian viscosity model.

A very important difference between Newtonian and non-Newtonian fluids in acoustic streaming velocity contours is observed. As Fig. 8 shows, outer boundary layer in non-Newtonian case has two split cores. These cores seem to be separated from each other by flow exited the channel. While for Newtonian one, as seen in fig 9, only one core at outer boundary layer is distinguished.

\section{CONCLUSIONS}

Effects of non-Newtonian viscosity, power law model on non-linear acoustofluidics are presented. A second-order perturbation theory is utilized. The following results are obtained:

1. Power-law viscosity model changes the zero and first order $\mathrm{x}$-velocities less than about $7 \%$ and $10 \%$, respectively.

2. It is shown that power-law model reduce the $x$-velocity by $60 \%$.

3. As shown zero and first order velocities for power-law and constant viscosity model are the same while a major difference in velocity pattern are observed by second-order velocity.

\section{REFERENCES}

Bernassau A. L., P. Glynne-Jones, F. Gesellchen, M. Riehle, M. Hill, and D. R. S. Cumming,(2014).Controlling acoustic streaming in an ultrasonic heptagonal tweezers with application to cell manipulation, Journal of Ultrasonics, 54, 268-274.

Hoyos M., and A. Castro, (2013).Controlling the acoustic streaming by pulsed ultrasounds, Journal of Ultrasonics, 53, 70-76.

Leia H., D. Henrya, and H. Ben Hadida, (2011).Acoustic force model for the fluid flow under standing waves, Journal of Applied Acoustics, 72, 754-759.

Muller P. B., and B. Henrik, (2014).Numerical study of thermoviscous effects in ultrasoundinduced acoustic streaming in microchannels, Journal of Physical Review, 90, 043016:1-12.

Paridaens R., S. Kouidri, and F. Jebali Jerbi, (2013).Investigation on the generation mechanisms of acoustic streaming in a thermoacoustic prime mover, Journal of Cryogenics, 58, 78-84.

Peneleta G, M. Guedraa, V. Gusevb, and T. Devauxa, (2012).Simplified account of Rayleigh streaming for the description of nonlinear processes leading to steady state sound in thermoacoustic engines, International Journal of Heat and Mass Transfer, 55, 60426053.

Sheua T. W. H., M. A. Solovchuk, A. W. J, Chena (2011).Thirietc M., an acoustics-thermal-fluid coupling model for the prediction of temperature elevation in liver tumor, International Journal of Heat and Mass Transfer, 54, 4117-4126.

Solovchuk Maxim A., W. H. Sheu Tony, Thiriet Marc , and W. L. Lin, (2013).On a computational study for investigating acoustic streaming and heating during focused ultrasound ablation of liver tumor, Journal of Applied Thermal Engineering, 56, 62-76.

Tajik B., A. Abbassi, M. Saffar-Avval, A.Abdullah, and H. Mohammad-Abadi, (2013).Heat transfer enhancement by acoustic streaming in a closed cylindrical enclosure filled with water, International Journal of Heat and Mass Transfer, 60, 230-235.

Wadaa Y., D. Koyamab, K. Nakamuraa, (2014).Acoustic streaming in an ultrasonic air pump with three-dimensional finite-difference time-domain analysis and comparison to the measurement, Journal of Ultrasonics, 54, 21192125. 